\title{
Comparison of Different Digital Modulation Techniques in LTE System using OFDM AWGN Channel: A Review
}

\author{
Swati Sharma \\ M.Tech, \\ GNDU, R C, Gurdaspur
}

\author{
Harjit Singh \\ Assistant Professor, \\ GNDU, RC, Gurdaspu
}

\begin{abstract}
In this paper there is comparison of all the modulation techniques which are possible to be used in LTE systems in OFDM AWGN channel. , BPSK, QPSK, 16QAM and 64QAM, so as to get the best one out of them on the basis of comparison parameters, bit error rate and signal to noise ratio because selection of adequate and suitable modulation technique is of utmost importance in wireless communication system .The paper gives modulation technique with least bit error rate and observes weather it is able to provide the required data rate in LTE systems.
\end{abstract}

\section{Keywords}

BER, SNR, LTE, LTE-A, BPSK, QPSK, QAM

\section{INTRODUCTION}

Now a days, we are living in the era of mobile data evolution. With a large expansion of tablets ,mobile phones, computers ,laptops ,users are demanding for such applications and services that are not only limited to mere voice but go far beyond it [11]. The rapid growth and development in services and applications such as social networking, video streaming, web browsing, music etc. has driven the mobile data technology towards next generation of wireless communication standards. LTE and LTE-A have developed to deliver such types of rich multimedia applications with the objectives to provide improved system capacity, higher data rates, low latency and reduced operating costs.LTE and LTEA are the representatives of the fourth generation standards or can be called as evolution for the third generation [2] . OFDM and SC-FDMA are the major parts of present wireless standards, LTE and LTE-A.OFDM was first developed in 1960 [12]. OFDM is the dominant technology in LTE and LTE-A systems because of its features, for example ,robustness to multipath fading channel, high spectral efficiency and ease of implementation [10] . OFDM can be implemented by using digital as well as analog modulation techniques. But, Digital modulation techniques offer, additional information-carrying capacity, improved data security, compatibility with digital data services, improved quality communication, swift system availability as well as RF spectrum sharing to accommodate more and more

Services [1]. Thus digital modulation techniques are being preferred specially in high data rate systems such as LTE, LTE-A. Different digital modulation techniques used in LTE and LTE-A are BPSK, QPSK, 16 QAM and 64 QAM. The selection of the best modulation technique which is to be used , depends upon SNR, BER ,cost effectiveness and ability to provide enhanced data rates [1]. In this paper we compared different modulation techniques in OFDM AWGN channel on the basis of two parameters, SNR and BER.

\section{OFDM}

Orthogonal frequency division multiplexing (OFDM) is very efficient, promising and reliable technique in today's high data rate wireless communication system. OFDM uses the concept of multicarrier modulation where each of the signal is broken into several new signals which are modulated to different frequency channels and then data is received and combined on multiple channels at the receiver [2]. In OFDM different channels are orthogonal to each other [9] and known as subcarriers .Condition of orthogonality is:

$$
\int \cos (2 \pi n f t) \cos (2 \pi m f t) d t=0
$$

$$
(\mathrm{m} \neq \mathrm{n})
$$

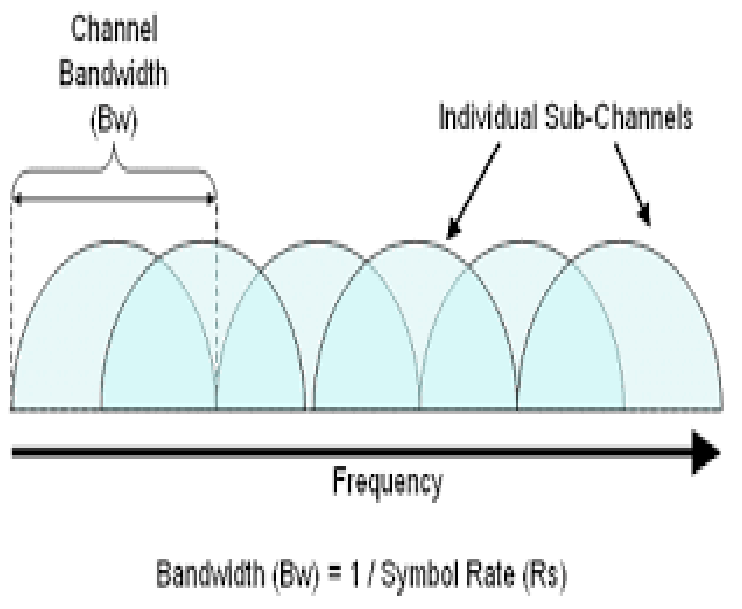

Figure 1. Orthogonal frequency division multiplexing [2]

\section{MODULATION TECHNIQUES}

Different modulation techniques that can be used in the LTE systems are given below:

\subsection{BPSK}

Phase shift keying is the digital modulation technique in which data is transmitted by changing the phase of the carrier wave. BPSK is the simplest form of the PSK (which is also termed as 2PSK) modulation in which two phases are separated by 180 . The general form of equation is given by [9]:

$s_{n}(t)=\sqrt{\frac{2 E_{b}}{T_{b}}} \cos \left(2 \pi f_{c} t+\pi(1-n)\right), n=0,1$.

Its constellation diagram is given by: 


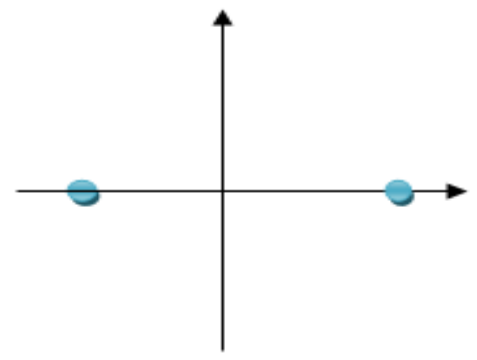

Figure 2. Constellation size example of $\operatorname{BPSK}[9,11]$

\subsection{QPSK}

Another digital modulation technique is higher order PSK that transmits 2 bits/symbol simultaneously by using a four level phase state [4], by selecting one of the four carrier phase shifts of $0, \pi / 2, \pi$ and $3 \pi / 2$, where each phase shift corresponds to bits of $00,01,10,11$. Thus double information is carried by using the same bandwidth. Mathematically, it is given as

$s_{n}(t)=\sqrt{\frac{2 E_{s}}{T_{s}}} \cos \left(2 \pi f_{c} t+(2 n-1) \frac{\pi}{4}\right), \quad n=1,2,3,4$.

Its constellation diagram is given by

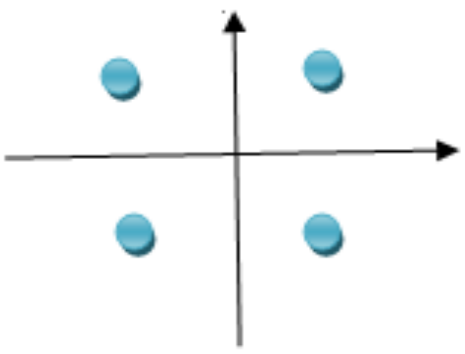

Figure 3. Constellation diagram for QPSK [11]

\subsection{QAM}

QAM is similar to the PSK apart from the fact that amplitude can also vary with phase in other words two different signals are to be transmitted on the same carrier frequency. QAM can be classified into different techniques such as 8QAM, 16QAM, 64QAM etc. Higher order QAM gives more points within the constellation, thus are capable to transmit more bits per symbol, and this to enable data to be transmitted in a much smaller bandwidth $[1,4]$.

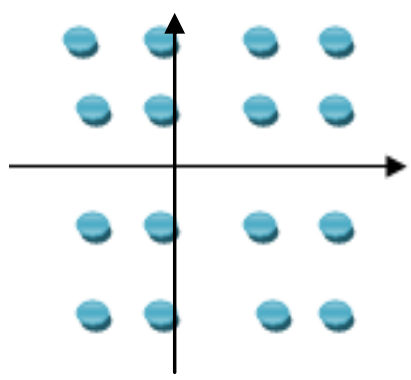

Figure 4. Constellation size of 16 QAM [11]

\section{AWGN CHANNEL}

A radio channel is simply an electromagnetic media between the transmitter and the receiver. The widely used channel model is the Gaussian channel, which is generally called the additive white Gaussian noise (AWGN) channel. The AWGN channel is very simple and usually it is considered as the starting point to develop the basic system for performance evaluation [8]. AWGN channel model is widely used in studying OFDM. In this model there is only linear addition of white noise with a constant spectral density and Gaussian distribution of amplitude. The model does not consider fading, frequency selectivity, interference etc. [10]. Although it is not much suitable for most of the terrestrial links yet being used for providing simple and controlled mathematical models to study the basic behaviour of a system in the absence of the above mentioned factors.

\section{SYSTEM SET-UP}

For comparison of different modulation techniques in OFDM AWGN channel is used. In LTE system MATLAB is used as simulator and two parameters are used, SNR (signal to noise ratio) and BER (bit error rate) .SNR within OFDM is considered as ratio of signal margin to the noise level. If ratio is higher than 1:1 then it is the indication of more signal than that of the noise. Signal-to-noise ratio is defined as the power ratio between a signal (meaningful information) and the background noise (unwanted signal) [2]:

$\mathrm{SNR}=\mathrm{P}($ signal $) / \mathrm{P}$ (noise) $[13]$

Where, $\mathrm{P}$ is an average power. Also bit error rate is the ratio of number of errors and number of bits sent. Can be explained By using formula:

$\mathrm{BER}=$ number of bits sent in error / total number of bits sent. [9]

There are some specifications used in simulation environment:

Table 1. Work Specifications in Simulation Work

\begin{tabular}{|c|c|}
\hline Parameters & Values \\
\hline Number Of (M)subcarriers & 2048 \\
\hline Guard interval samples(M/4) & 512 \\
\hline Guard interval length & $2.5 \mathrm{us}$ \\
\hline & \\
\hline Modulation level (q) for : & \\
BPSK & 2 \\
QPSK & 4 \\
16QAM & 16 \\
64QAM & 64 \\
\hline
\end{tabular}

\section{RESULTS \&DISCUSSION}

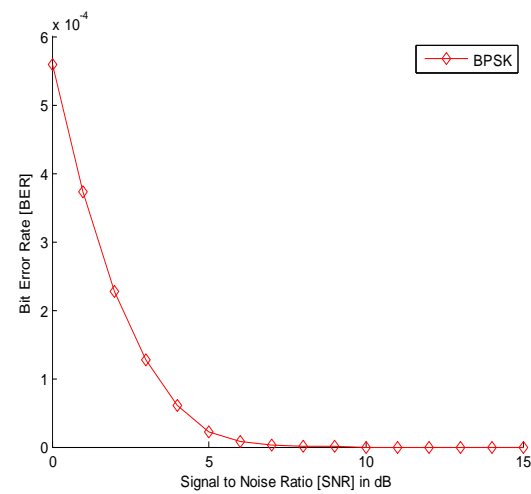

Figure [5] BPSK 
It is clear from the figure that in BPSK, BER curve approaches to zero as SNR appraoches to 6 . Thus clearly, the performance is quite good if we use BPSK modulation technique, taking error rate into consideration. But if we use BPSK we will not be able to send data on higher rates because of rarer constellation in BPSK. This fact makes BPSK imapropriate to be used in applications where very high data rates are required

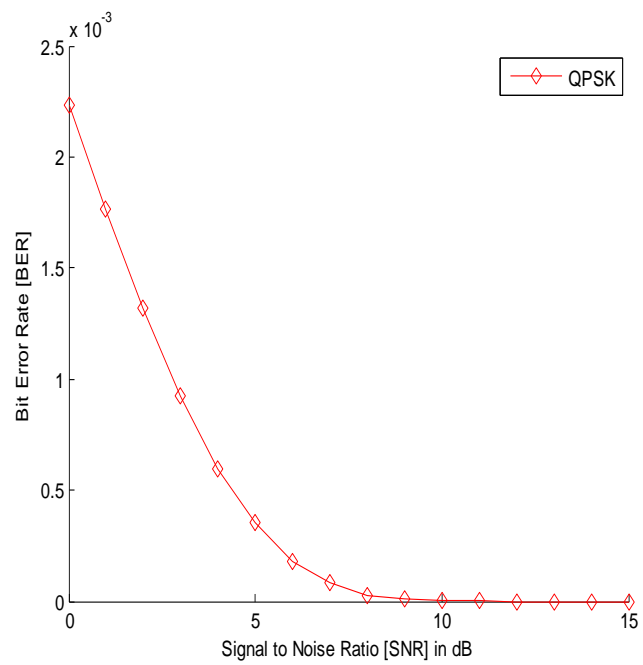

Figure[6] QPSK

In Figure[6] in QPSK , BER curve approaches to zero when SNR approaches to 8 . The curve shows QPSK gives more error rate than that of the BPSK. So, the applications where noise and errors are the major parametres to be considered, QPSK is preferred over BPSK .

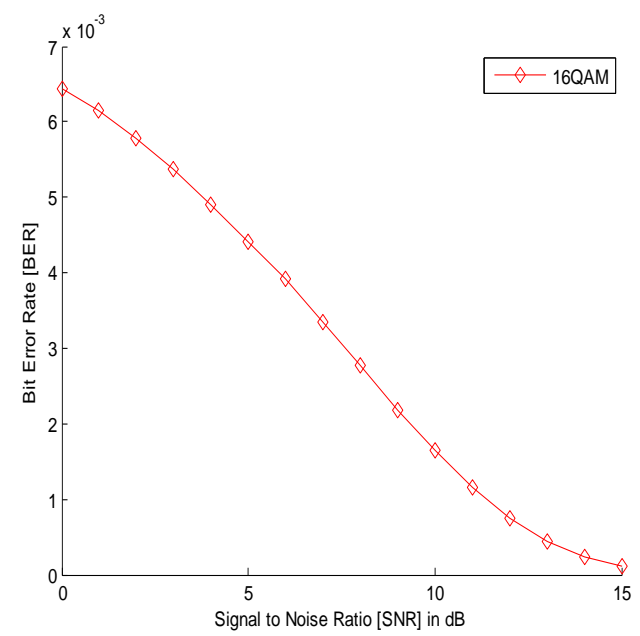

Figure [7] 16QAM

From figure [7] it can be seen that in 16QAM , BER curve approaches to zero when SNR approaches to 15 . It shows that 16 QAM will not be used where low error rate is prioritised .

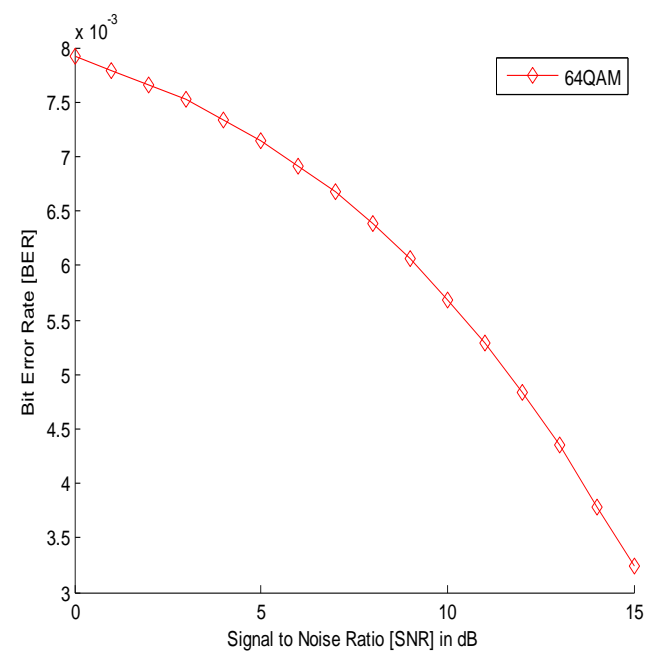

Figure[ 8] 64QAM

IN 64 QAM BER does not reach to 0 even if SNR is 15 . Thus the usage of 64QAM modulation technique will be inappropriate . But also 64 QAM is able to send data at very higher data rates because of denser constellation .

From figure it is very clear that:

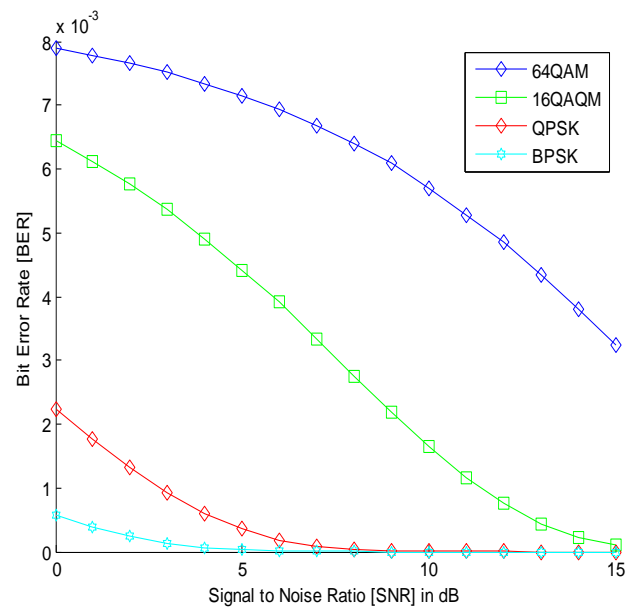

Figure [9] comparison of modulation techniques

From figure [9] it is very clear that:

\section{BERBPSK $<$ BEROPSK $<$ BER16QAM $<$ BER64QAM}

Apparently, BPSK gives least error rate and with the increase in order of modulation, Bit error rate goes on increasing with SNR. 64 QAM has the maximum error rate. Thus on the basis of robustness to the noise BPSK is the best technique to implement but in the systems such as LTE and LTE-A higher data rates are required as we can see from the table [11] - 
Table2. Theoratical Peak Data Rates

\begin{tabular}{|l|l|}
\hline Technology & $\begin{array}{l}\text { THEORATICAL PEAK DATA } \\
\text { RATES(At low mobility) }\end{array}$ \\
\hline GSM & $9.6 \mathrm{kbps}$ \\
\hline IS-95 & $14.4 \mathrm{kbps}$ \\
\hline GPRS & $171.2 \mathrm{kbps}$ \\
\hline EDGE & $473 \mathrm{kbps}$ \\
\hline CDMA-2000 & $307 \mathrm{kbps}$ \\
\hline WCDMA & $1.92 \mathrm{kbps}$ \\
\hline HSDPA(Rel-5) & $14 \mathrm{Mbps}$ \\
\hline HSPA+(Rel-6) & $84 \mathrm{Mbps}$ \\
\hline LTE(REL 8) & $300 \mathrm{Mbps}$ \\
\hline LTE-ADVANCED & \\
\hline
\end{tabular}

64QAM is capable of transmitting higher data rates because of denser constellation but, it is very prone to noise and errors .so, such techniques should be implemented that 64 QAM can be used with comparatively lesser error rate, for example bit level scrambling and turbo coding .

\section{CONCLUSION}

The paper has concluded that higher is the order of digital modulation scheme more will be the bit error rate. But in LTE system more data rates are required which are only possible to be achieved with higher order of modulation. Thus in LTE system higher order of modulation should be implemented with bit error rate reduction techniques such as turbo coding, equalisation etc. So that higher data rates can be achieved with lesser bit error rate.

\section{REFERENCES}

[1] Charles U. Ndujiuba, Oluyinka Oni and Augustus E. Ibhaze, "Comparative Analysis of Digital Modulation Techniques in LTE 4G Systems", Journal of Wireless Networking and Communications, 5(2), 60-66, 2015.

[2] Santosh M Nejakar and Prabhu G Benakopand Sharanabasappa R R , "Orthogonal Frequency Division Multiplexing Modulation Scheme for 4G/5G Cellular Network", European Journal of Advances in Engineering and Technology, 2(3),46-50,2015.

[3] Patel Sneha Bhanubhai, Mary Grace Shajan, and Upena D. Dalal, "Performance of Turbo Encoder and Turbo Decoder for LTE", International Journal of Engineering and Innovative Technology (IJEIT), Volume 2, Issue 6, October 2012.

[4] Sara Riahi 1, Ali El Hore2 and Jamal El Kafi," Performance study of the OFDM modulation for the use in Wireless communication Systems of the 4G,'International Research Journal of Engineering and Technology (IRJET), Volume: 02, 2013.

[5] Kovalenko, A.I.; Zhukov, R.V.; Shelkovnikov and B.N., "Features of long term evolution technology and comparative analysis of noise stability for different kinds of modulation," $19^{\text {th }}$ International Crimean Conference in Microwave \& Telecommunication Technology, vol.5, no.10, pp.235-236, Sept. 2009

[6] T.Latha,S. Priyadarshini "Effective Communication in LTE-A Using Link Adaptation Technique", IEEE Sponsored 2nd International Conference on Innovations in information, Embedded and Communication systems ( ICllECS ), 2015.

[7] Anuradha and Naresh kumar, "BER analysis of conventional and wavelet based OFDM in LTE using different modulation techniques", Proceedings of 2014, RAECS UIET Panjab University Chandigarh, $06-08$ March, 2014.

[8] Abhishek Katariya, Amita Yadav and Neha Jain , "Performance Elevation Criteria for OFDM under AWGN Fading Channel using IEEE 802.11a “ , International Journal of Soft Computing and Engineering (IJSCE), Volume-1, Issue-3, July 2011 .

[9] Mr. Mahendra Kumar Bairwa, Dr. Mahesh Porwal, "BER Performance of OFDM System over AWGN channel with Different Modulation Schemes", International Journal of Engineering and Technical Research (IJETR), Volume-2, Issue-8, August 2014.

[10] Anurag Pandey and Sandeep Sharma, BER Performance of OFDM System in AWGN andRayleigh Fading Channel "International Journal of Engineering Trends and Technology (IJETT), Volume 13 Number 3, Jul 2014.

[11] Dr Houman Zarrinkoub, Understanding LTEWith MATLAB, john wiley and sons ltd., 2014.

[12] Navjot Kaur and Er. Neetu Gupta , "Simulation and Analysis of OFDM and SC-FDMA with STBC using Different Modulation Techniques" ,International Journal of Advanced Research in Computer Engineering \& Technology (IJARCET), Volume 4 ,Issue 11, November 2015 .

[13] Pravin K. Patil "Role of Contributing Factors MIMOOFDM in 4G-LTE Wireless Transmission Technologies from Technical Perspective ",International Journal of Advanced Research in Computer and Communication Engineering,Vol. 2, Issue 7, July 2013. 